\title{
Evaluation de la diversité des diptères hématophages dans une clairière marécageuse du Gabon à I'aide des pièges Vavoua et Nzi
}

\author{
R.C. Zinga Koumba 1,2* J. Bouyer 3,4 \\ J.F. Mavoungou ${ }^{1,5}$ G.L. Acapovi Yao ${ }^{6}$ \\ L. Kohagne Tongué 7 O.A. Mbang Nguema ${ }^{1,5}$ \\ K.P.O. Ondo ${ }^{1}$ S. Mutambwe ${ }^{2}$
}

\begin{abstract}
Mots-clés
Glossina - Stomoxys - Tabanidae Piège Vavoua - Piège Nzi - Forêt Marécage - Gabon.
\end{abstract}

\begin{abstract}
Résumé
L'objectif de ce travail a été d'étudier la diversité des insectes hématophages présents dans les clairières forestières marécageuses (bai) du Gabon à l'aide de deux types de pièges : le Vavoua et le Nzi. Vingt pièges (10 de chaque type) ont été placés à l'intérieur et autour du baï de Momba pendant sept jours consécutifs durant le mois de juin 2012. Dans chacun de ces deux biotopes, cinq paires de pièges (un Vavoua et un Nzi espacés I'un de l'autre de $30 \mathrm{~m}$ environ) ont été posés à intervalles de 250-400 m. Au total, 662 glossines, 42 tabanides et 20 stomoxes ont été collectés. Le piège Vavoua a capturé de nombreux stomoxes et glossines, alors que le piège Nzi a capturé davantage de tabanides. Compte tenu de la faible durée de capture, une prolongation du suivi de ces insectes a été par la suite effectuée pour préciser leur importance relative et leur dynamique saisonnière.
\end{abstract}

\section{INTRODUCTION}

Le piégeage des diptères hématophages, en l'occurrence les tabanides, les stomoxes et les glossines, constitue un moyen de capture économique permettant d'obtenir des échantillons de population plus représentatifs que ceux obtenus par capture au filet (12). Par ailleurs, le piégeage représente un outil efficace d'étude, de surveillance, de protection et de lutte écologique contre les vecteurs (8). Enfin, par sa simplicité, le piégeage constitue l'une des méthodes les plus adaptées pour assurer la participation communautaire et le respect du milieu $(6,13,19)$.

Les diptères hématophages ont un rôle extrêmement important en santé humaine et animale (24). En effet ces insectes, en raison de

1. Institut de recherche en écologie tropicale, BP 13354, Libreville, Gabon.

2. Ecole régionale post-universitaire d'aménagement et de gestion intégrés des forêts et territoires tropicaux, Kinshasa, R. D. Congo.

3. Cirad, UMR Contrôle des maladies animales exotiques et émergentes, Montpellier, France.

4. Institut sénégalais de recherches agricoles, Laboratoire national d'élevage et de recherches vétérinaires, Dakar-Hann, Sénégal.

5. Université des sciences et techniques de Masuku, Franceville, Gabon.

6. Université Félix Houphouët-Boigny, UFR Biosciences, Abidjan, Côte d'Ivoire.

7. Association de promotion pour la lutte contre les parasitoses, Yaoundé, Cameroun.

* Auteur pour la correspondance

Tél. : + 24104404923 ; e-mail : zinga.koumba@yahoo.fr leur mode d'alimentation, représentent un fléau à la fois par leur nuisance directe (harcèlement et prédation) $(7,9)$ et par leur rôle de vecteur potentiel de divers agents pathogènes $(5,14,15,40)$. Ils sont impliqués dans la transmission biologique et/ou mécanique de nombreuses maladies comme la fièvre de la vallée du Rift, l'anaplasmose bovine et les trypanosomoses africaines (humaine et animale) $(1,2,16)$. Il est donc important d'en étudier la diversité.

De nombreux travaux ont évalué l'efficacité des techniques de capture des tabanides, des glossines et des stomoxes, et les pièges Nzi et Vavoua se sont révélés très efficaces, respectivement pour les vecteurs mécaniques et pour les glossines $(8,16,18,28)$. Des études comparatives de l'efficacité des pièges ont été effectuées en Afrique de l'Ouest et plus rarement en Afrique de l'Est (3, 4, 15, 22, 28, 35, 36). En revanche, les connaissances concernant la diversité des insectes hématophages dans les clairières forestières du Gabon sont rares et anciennes. L'étude présentée ici a eu pour objectif d'identifier les principales espèces de diptères hématophages dans deux biotopes ne présentant pas le même degré d'ouverture de la végétation. L'utilisation simultanée de plusieurs types de piège a permis de réaliser des inventaires plus complets de la diversité rencontrée. Toutefois, l'efficacité des deux types de pièges utilisés n'a pas pu être formellement comparée car nous n'avons pas réalisé de carré latin. De plus, en raison de sa durée limitée, il a été nécessaire de poursuivre l'étude à différentes saisons pour compléter l'inventaire des espèces présenté ici (40). 


\section{MATERIEL ET METHODES}

\section{Zone d'étude}

L'étude a été réalisée au baï (clairière forestière marécageuse) de Momba $\left(0,027^{\circ}\right.$ de lat. $\mathrm{N}$ et $13,424^{\circ}$ de long. E), situé dans le nordest du Gabon à environ 160 kilomètres de la ville de Makokou, et dans la forêt primaire qui l'entoure (figure 1). Le climat de cette région est de type équatorial, caractérisé par la double alternance de saisons sèches (de mi-décembre à mi-mars, puis de mi-juin à mi-septembre) et de saisons des pluies (de mi-mars à mi-juin, puis de mi-septembre à mi-décembre). La température moyenne est de $24^{\circ} \mathrm{C}$ avec un minimum de $21,7^{\circ} \mathrm{C}$ en juillet et un maximum de $25^{\circ} \mathrm{C}$ en avril. Les amplitudes thermiques annuelles et journalières sont faibles (environ $3,3^{\circ} \mathrm{C}$ ). La pluviométrie annuelle moyenne est de l'ordre de $1700 \mathrm{~mm}$, les plus fortes précipitations ayant lieu en octobre et novembre, puis de mars à mai (38). Le paysage est caractérisé par une végétation dominée par des Cyperaceae, des Poaceae et des Rubiaceae. La grande faune est représentée par plusieurs espèces de mammifères dont l'éléphant (Loxodonta africana cyclotis), le buffle (Syncerus caffer nanus) et le sitatunga (Tragelaphus spekei).

\section{Capture des mouches hématophages}

Deux types de pièges ont été utilisés : le piège Vavoua (21), qui permet la capture des glossines riveraines et des stomoxes, et le piège Nzi $(15,27)$, qui est particulièrement efficace pour la capture des tabanides.

Deux biotopes ont été prospectés : d'une part, la lisière du bai (milieu ouvert) qui fait moins d'un kilomètre carré, où les pièges ont été posés sur environ un kilomètre et, d'autre part, la forêt primaire qui le borde (milieu fermé), étudiée sur une profondeur d'environ $1,25 \mathrm{~km}$. Dans chacun de ces biotopes, 10 pièges $(5$ Vavoua et $5 \mathrm{Nzi}$ ) ont été posés par groupes de deux pièges (un Vavoua et un Nzi, espacés d'environ $30 \mathrm{~m}$ ). Les deux pièges de chaque groupe constituaient chacun un point de capture d'un site

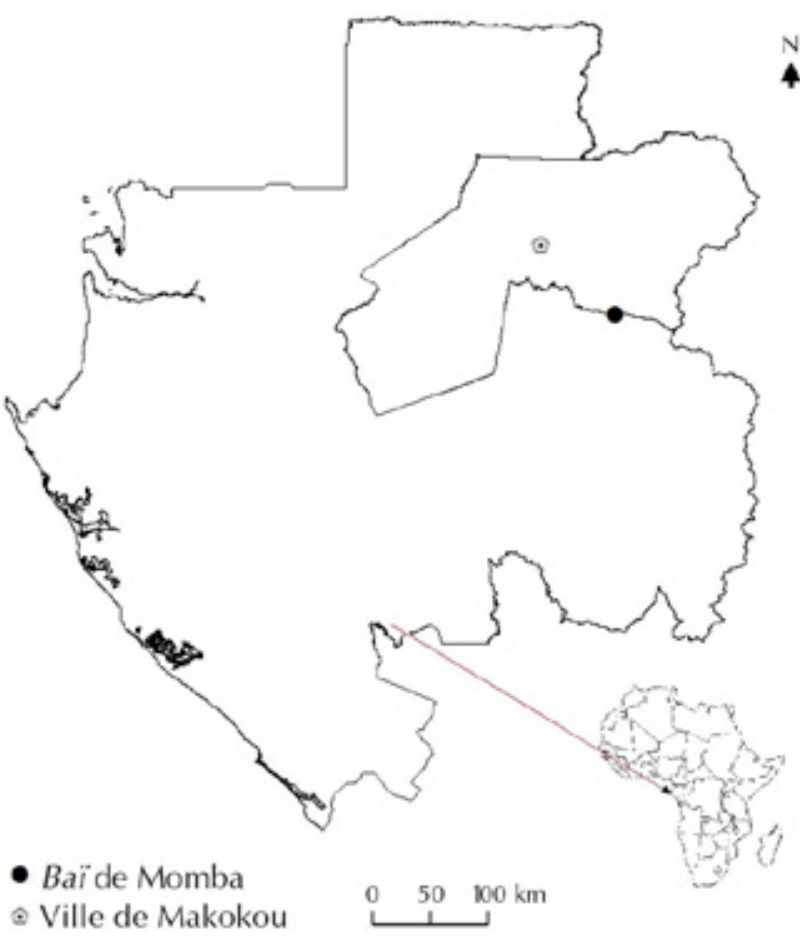

Source : laboratoire de cartographie de l'IRET. Réalisation : Maroundou A. P. 15/04/2014

Figure 1 : localisation du baï de Momba au Gabon. de piégeage, ces sites étant distants des autres sites de piégeage de $250 \mathrm{~m}$ à $400 \mathrm{~m}$. Les pièges Nzi posés en lisière du baï ont été orientés vers la forêt.

La durée de piégeage a été de sept jours consécutifs et l'effort global de capture dans la journée a été de dix heures, les pièges ayant été activés le matin avant $7 \mathrm{~h}$ et relevés le soir après $17 \mathrm{~h}$. Lors de la relève des pièges, les cages de capture ont été étiquetées avec le numéro du piège et ramenées au laboratoire temporaire aménagé pour la circonstance non loin du site d'étude, à environ deux kilomètres. Toutes les captures ont été réalisées en juin 2012, c'est-àdire pendant la période marquant la fin de la saison des pluies et le début de la saison sèche.

\section{Identification des insectes}

Au laboratoire, les glossines, stomoxes et tabanides ont été séparés des autres spécimens, puis dénombrés et identifiés sous une loupe binoculaire à l'aide des clés d'identification publiées par Oldroyd (30-33), Zumpt (41), Surcouf et Ricardo (37), Garros et coll. (17), Matyas (23), et à l'aide du logiciel d'identification des glossines (10).

\section{Analyse des données}

L'abondance de chaque espèce de mouche hématophage étudiée a été traduite par sa densité apparente par piège et par jour (DAP). Les DAP ont ensuite été analysées par des modèles linéaires généralisés à effets mixtes (librairie lme4 du logiciel gratuit R) (34) se basant sur une distribution de Poisson. Les paysages et types de pièges et leurs interactions ont été considérés comme des effets fixes, les sites de piégeages comme des effets aléatoires. Les analyses ont été effectuées à l'échelle des familles, puis des quelques espèces de glossines les plus abondantes. L'indice de diversité de Margalef a été calculé pour déterminer la diversité des espèces capturées par chacun des pièges dans les deux biotopes, selon la formule $\mathrm{D}=(\mathrm{S}-1) / \log \mathrm{N}$, avec $\mathrm{S}$ le nombre d'espèces et $\mathrm{N}$ le nombre d'individus récoltés $(1,2)$.

\section{RESULTATS \\ Densité apparente des insectes étudiés à l'échelle des familles}

Au total, dans les deux biotopes prospectés, 724 mouches piqueuses ont été capturées : 662 glossines, 42 tabanides et 20 stomoxes (tableau I). Le piège Vavoua a montré une affinité supérieure au piège Nzi pour la capture des glossines, la densité de ces dernières étant plus élevée dans la clairière qu'en forêt $\left(\mathrm{p}<10^{-3}\right)$. Quant au piège Nzi, il a capturé des tabanides en plus grand nombre. En ce qui concerne les stomoxes, seul le piège Vavoua en a capturé, et uniquement dans la clairière. Dans ces deux cas, les différences de densité entre les deux biotopes n'étaient pas significatives, en raison des faibles effectifs capturés (figure 2).

\section{Diversité spécifique en fonction du type de piège}

Les indices de Margalef représentant la diversité des espèces capturées par type de piège ont montré que le maximum d'espèces de mouches hématophages a été capturé avec le piège Vavoua (figure 3). Seules deux espèces de tabanides, Ancala fasciata et Tabanus dilutius, n'ont pas été capturées par le piège Vavoua, alors que le piège Nzi n'a capturé aucun stomoxe. Les tabanides ont présenté la biodiversité la plus importante, suivie par les glossines puis les stomoxes. Chez les glossines, Glossina palpalis palpalis a représenté plus de la moitié des captures à elle seule. 


\section{Tableau I}

Densités apparentes ${ }^{1}$ par piège ${ }^{2}$ et par jour des glossines, stomoxes et tabanides, et signification des différences entre biotopes

\begin{tabular}{|c|c|c|c|c|}
\hline Taxon & Clairière & Forêt & Global & Différence entre biotopes \\
\hline Glossina frezili & $0,37(0,97)$ & $0,03(0,17)$ & $0,20(0,71)$ & $*$ \\
\hline Glossina fusca congolensis & $1,31(2,86)$ & $0,17(0,45)$ & $0,74(2,11)$ & $* * *$ \\
\hline Glossina fuscipes fuscipes & $0,26(0,95)$ & $0,26(0,74)$ & $0,26(0,85)$ & NS \\
\hline Glossina nashi & $2,00(4,92)$ & $0,26(0,89)$ & $1,13(3,62)$ & $* * *$ \\
\hline Glossina palpalis palpalis & $5,91(9,88)$ & $2,09(3,58)$ & $4,00(7,63)$ & $* * *$ \\
\hline Glossina sp. & $0,66(1,41)$ & $0,26(0,61)$ & $0,46(1,10)$ & * \\
\hline Glossinidae & $10,51(18,06)$ & $3,06(4,61)$ & $6,79(13,61)$ & $* * *$ \\
\hline Stomoxys inornatus & $0,09(0,37)$ & - & $0,04(0,27)$ & NS \\
\hline Stomoxys niger niger & $0,34(1,30)$ & - & $0,17(0,93)$ & NS \\
\hline Stomoxys omega & $0,14(0,85)$ & - & $0,07(0,60)$ & NS \\
\hline Stomoxyinae & $0,57(2,10)$ & - & $0,29(1,50)$ & NS \\
\hline Ancala fasciata & $0,14(0,55)$ & - & $0,07(0,39)$ & NS \\
\hline Chrysops longicornis & $0,11(0,53)$ & $0,14(0,36)$ & $0,13(0,45)$ & NS \\
\hline Chrysops silacea & - & $0,20(0,90)$ & $0,10(0,64)$ & NS \\
\hline Tabanus dilutius & $0,03(0,17)$ & - & $0,01(0,12)$ & NS \\
\hline Tabanus obscurefumatus & $0,03(0,17)$ & - & $0,01(0,12)$ & NS \\
\hline Tabanus par & $0,23(0,73)$ & - & $0,11(0,53)$ & NS \\
\hline Tabanidae & $0,57(1,70)$ & $0,34(0,94)$ & $0,46(1,37)$ & NS \\
\hline
\end{tabular}

${ }^{1}$ Ecarts types entre parenthèses

${ }^{2}$ Ont été mesurés ici les glossines et les stomoxes capturés dans les pièges Vavoua et les tabanides capturés dans les pièges Nzi.

$* * * \mathrm{p}<10^{-3} ; * \mathrm{p}<0,05 ; \mathrm{NS}: \mathrm{p}>0,05$

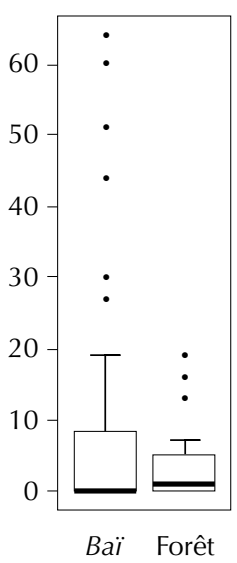

Figure 2 : de gauche à droite, densités apparentes par piège et par jour des glossines (piège Vavoua), des stomoxes (piège Vavoua) et des tabanides (piège Nzi) dans la clairière marécageuse de Momba et la forêt attenante.

\section{Composition et abondance spécifique des insectes en fonction du biotope}

Cinq espèces de glossines ont été capturées avec le piège Vavoua. Il s'agissait, par ordre d'importance décroissant, de G. p. palpalis (59 p. 100 des captures), G. nashi (17 p. 100), G. fusca congolensis (11 p. 100), G. fuscipes fuscipes (4 p. 100) et G. frezili (3 p. 100). Ces mêmes espèces ont été capturées par le piège Nzi, avec des abondances plus faibles. Sept pour cent des individus n'ont pas pu être identifiés.

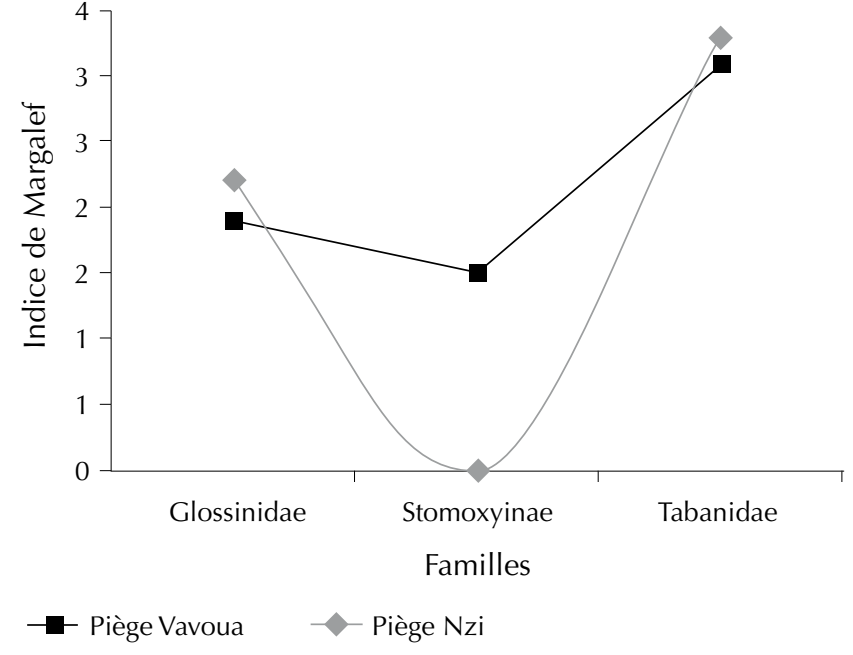

Figure 3 : densité d'insectes capturés selon le type de pièges.

Le tableau I présente la densité apparente moyenne par piège et par jour des différentes espèces en fonction du biotope et la signification des différences entre ces biotopes (résultats des modèles linéaires généralisés à effets mixtes). On peut noter que les captures ont été globalement significativement supérieures en clairière pour toutes les espèces de glossines.

Six espèces de tabanides (Ancala fasciata, Chrysops longicornis, C. silacea, Tabanus dilutius, T. obscurefumatus, et T. par) ont été capturées avec le piège Nzi contre quatre avec le piège Vavoua (C. longicornis, C. silacea, T. obscurefumatus et T. par). Seule 
l'espèce d'un individu n'a pas pu être identifiée. Aucune différence significative entre biotopes n'a été observée au niveau des espèces mais il était intéressant de constater que les Chrysops étaient présents en densité égale à supérieure en forêt, contrairement aux autres espèces, ce qui correspond bien à l'écologie comportementale de ces tabanides.

Les stomoxes capturés avec le piège Vavoua ont été identifiés et appartenaient aux espèces Stomoxys niger niger (60 p. 100), S. omega (25 p. 100) et $S$. inornatus (15 p. 100). Aucune différence significative entre biotopes n'a été observée.

\section{DISCUSSION}

Dans cette étude un travail préliminaire a été réalisé sur l'inventaire spécifique des glossines, stomoxes et tabanides dans les clairières forestières marécageuses au Gabon. Les glossines étaient largement prédominantes et capturées en densité trois fois supérieure en lisière de clairière par rapport à l'intérieur de la forêt, ce qui souligne le risque de transmission trypanosomienne associé à ce type de site. Cette répartition hétérogène peut être liée aux paysages. En effet, la forêt primaire est caractérisée par de très grands arbres (jusqu'à $50 \mathrm{~m}$ de haut et plus de $2 \mathrm{~m}$ de diamètre) dont les cimes forment une canopée qui obscurcit le sous-bois et atténue considérablement la température. D'autre part, la visibilité des pièges, et donc leur efficacité, y est réduite. En revanche, la clairière présente une végétation plus basse et les températures y sont relativement plus élevées en relation avec un temps d'éclairement plus important. Nos résultats corroborent ainsi des études conduites par Mavoungou et coll. (25) dans la réserve de biosphère d'Ipassa-Makokou au Gabon qui montrent que le maximum de captures d'insectes hématophages est observé dans les milieux ouverts.

Les résultats obtenus dans cette étude indiquent que les pièges Vavoua sont qualitativement supérieurs pour piéger les glossines et les stomoxes dans ces biotopes, alors que les pièges Nzi sont plus efficaces pour les tabanides. Ces résultats corroborent ceux obtenus par Gilles et coll. (18), et par Lindh et coll. (22). Cependant, en l'absence de rotation des pièges entre sites (carré latin), il n'est pas possible de comparer les efficacités quantitativement, même si les différences observées étaient significatives, en raison du possible effet « site de capture ». L'utilisation de deux types de piège a permis d'augmenter le nombre d'espèces piégées, le Nzi n'ayant pas capturé de stomoxes mais ayant piégé plus de tabanides que le Vavoua. Ces résultats confirment l'efficacité du piège Nzi pour la capture des tabanides, et du piège Vavoua pour la capture des stomoxes et des glossines dans les clairières forestières (27).

La faible abondance des stomoxes et des tabanides dans cette étude pourrait s'expliquer par l'absence de pluies (l'étude a été réalisée en saison sèche). Ces résultats sont similaires à ceux obtenus par Mavoungou et coll. $(24,26)$ qui montrent l'importance des précipitations sur l'augmentation de la taille des populations des diptères hématophages. Dans d'autres pays, cependant, les densités de tabanides et de stomoxes sont maximales en début de saison sèche $(20)$.

Les travaux conduits par Mavoungou et coll. $(24,26)$ à Makokou au Gabon ont révélé la présence de sept espèces de stomoxes. Dans notre étude, trois espèces seulement ont été capturées. Cela traduit-il une richesse spécifique apparemment plus faible au baï de Momba, au moins pendant la saison sèche ? On ne peut cependant pas conclure pour le moment car l'étude a été effectuée pendant seulement une semaine et à raison de 10 heures par jour.
Les faibles abondances de tabanides, en particulier du genre Chrysops, pourraient s'expliquer par une faible attractivité des pièges utilisés. Des études conduites par Noireau et coll. (29), et Caubère et Noireau (11) en République du Congo, et par Wandji et coll. (39) au Cameroun montrent que les meilleures captures pour ces espèces s'obtenaient au filet en présence d'un feu de bois, notamment pour $C$. silacea et $C$. dimidiata. Toutefois, ces deux espèces ont été abondantes en forêt, ce qui corrobore les résultats de Noireau et coll. (29), et Caubère et Noireau (11).

Une prolongation du suivi de ces insectes a été effectuée pour préciser leur importance relative et leur dynamique saisonnière (40).

\section{Remerciements}

Ce travail a été réalisé grâce à l'appui financier, institutionnel et logistique de l'Union européenne via le projet ACP-Forenet, l'Institut de recherche en écologie tropicale (Iret/Cenarest), l'Ecole régionale post-universitaire d'aménagement et de gestion intégrée des forêts et territoires tropicaux (Eraift) à travers la bourse Ceeac/ Pacebo, et la bourse de Wallonie-Bruxelles International. Nous remercions Drs A. Ngomanda, J. Okouyi Okouyi et N. Engone Obiang, ainsi que MM. K. Henga Botsika Bobe, C. Mikala, E. Nzengue et P. Ondo Okama pour leurs précieux et rigoureux conseils. Nous remercions également A.P. Maroundou pour la réalisation de la carte du baï de Momba, et N. Emba Yao et S. Essasou Badji pour leur aide technique sur le terrain.

\section{BIBLIOGRAPHIE}

1. ACAPOVI G.L., YAO Y., N'GORAN E., DIA M.L., DESQUESNES M., 2001. Relative abundance of tabanids in the savanna regions of Côte d'Ivoire. Rev. Elev. Méd. Vét. Pays Trop., 54: 109-114. [in French]

2. ACAPOVI-YAO G.L., 2005. Identification et bioécologie des tabanidés, vecteurs mécaniques potentiels de la transmission de la trypanosomose bovine dans les régions de savanes en Côte-d'Ivoire (Odienné et Korhogo). Thèse Doct., Université d'Abidjan, Côte-d'Ivoire.

3. AMSLER S., FILLEDIER J., 1994. Attractiveness for Tabanidae of the meta-cresol/octenol association: results in Burkina Faso. Rev. Elev. Méd. Vét. Pays Trop., 47: 93-96. [in French]

4. AMSLER S., FILLEDIER J., 1994. Comparison of different systems for collecting tsetse and horse flies with two types of trap. Rev. Elev. Méd. Vét. Pays Trop., 47: 387-396. [in French]

5. BALDACCHINO F., MUENWORN V., DESQUESNES M., DESOLI F., CHAROENVIRIYAPHAP T., DUVALLET G., 2013. Transmission of pathogens by Stomoxys flies (Diptera, Muscidae): a review. Parasite, 20: 26.

6. BAUER B., AMSLER-DELAFOSSE S., KABORE I., KAMUANGA M., 1999. Improvement of cattle productivity through rapid alleviation of African trypanosomosis by integrated disease management practices in the agropastoral zone of Yalé, Burkina Faso. Trop. Anim. Health Prod., 31: 89-102.

7. BISHOPP F.C., 1913. The stable fly (Stomoxys calcitrans L.), an important livestock pest. J. Econ. Entomol., 6: 112-126.

8. BOUYER J., DESQUESNES M., KABORE I., DIA M.L., GILLES J., YONI W., CUISANCE D., 2005. Le piégeage des insectes vecteurs. Santé animale en Afrique de l'Ouest, fiche $\mathrm{n}^{\circ} 20$. Bobo-Dioulasso, Burkina Faso, Cirdes.

9. BOUYER J., GRIMAUD Y., PANNEQUIN M., ESNAULT O., DESQUESNES M., 2011. Importance épidémiologique et contrôle des stomoxes à la Réunion. Bull. épidémiol., nº spécial DOM-TOM, 43 : 5358 .

10. BRUNHES J., CUISANCE D., GEOFFROY B., HERVY J.P., 1998. LeS glossines ou mouches tsé-tsé. Logiciel d'identification et d'enseignement. Montpellier, France, Orstom.

11. CAUBERE P., NOIREAU F., 1991. Effect of attraction factors on the sampling of Chrysops silacea and C. dimidiata (Diptera: Tabanidae), vectors of Loa loa (Filaroidea: Onchocercidae) filariasis. J. Med. Entomol., 28: $263-265$ 
12. CHALlieR A., EYRAUD M., LAFAYE A., LAVEISSIERE C., 1977. Amélioration du rendement du piège biconique pour glossines (Diptera, Glossinidae) par l'emploi d'un cône inférieur bleu. Cah. ORSTOM, Sér. Entomol. Méd. Parasitol., 15 : 283-286.

13. CUISANCE D., 1989. Le piégeage des tsé-tsé. Maisons-Alfort, France, IEMVT, p. 172. (Coll. Etudes et synthèses, $\mathrm{n}^{\circ} 32$,

14. CUISANCE D., BARRE N., DE DEKEN R., 1994. Ectoparasites des animaux : méthodes de lutte écologique, biologique, génétique et mécanique. Rev. Sci. Tech. Off. Int. Epizoot., 13 : 1305-1356.

15. DESQUESNES M., DIA M.L., ACAPOVI G.L., YONI W., FOIL L., PIN R., 2005. Les vecteurs mécaniques des trypanosomoses animales: généralités, morphologie, biologie, impacts et contrôle. Identification des espèces les plus abondantes en Afrique de l'Ouest. Bobo-Dioulasso, Burkina Faso, Cirdes, 68 p.

16. DIA M.L., DESQUESNES M., HAMADOU S., BOUYER J., YONI W., GOURO A.S., 2008. Piège Tétra: Evaluation d'un modèle de petite taille pour la capture des vecteurs des trypanosomoses animales. Rev. Méd. Vét., 159 : 17-21.

17. GARROS C., GILLES J., DUVALLET G., 2004. Un nouveau caractère morphologique pour distinguer Stomoxys calcitrans et S. niger (Diptera : Muscidae). Comparaison de populations de l'île de La Réunion. Parasite, $11: 329-332$.

18. GILLES J., DAVID J.F., DUVALLET G., DE LA ROCQUE S., TILLARD E., 2007. Efficiency of traps for Stomoxys calcitrans and Stomoxys niger niger on Reunion Island. Méd. Vét. Entomol., 21 : 65-69.

19. KAMUANGA M., SIGUE H., SWALLOW B., BAUER B., D'IETEREN G., 2001. Farmers' perceptions of the impacts of tsetse and trypanosomosis control on livestock production: evidence from southern Burkina Faso. Trop. Anim. Health Prod., 33: 141-153.

20. KONE N., N'GORAN E.K., SIDIBE I., KOMBASSERE A.W., BOUYER J., 2011. Spatio-temporal distribution of tsetse and other biting flies in the Mouhoun River Basin, Burkina Faso. Med. Vet. Entomol., 25: 156-168.

21. LAVESSIERE C., GREBAUT P., 1990. Recherches sur les pièges à glossines (Diptera : Glossinidae). Mise au point d'un modèle économique : le piège "Vavoua ». Trop. Med. Parasitol., 41 : 185-192.

22. LINDH J.M., TORR S.J., VALE G.A., LEHANE M.J., 2009. Improving the cost-effectiveness of artificial visual baits for controlling the tsetse fly Glossina fuscipes fuscipes. PloS Negl. Trop. Dis., 3. DOI: 10.1371/ journal.pntd.0000474

23. MATYAS P.A., 1958. Tabanidae. In: Fauna Hungariae, vol. 16, Diptera I, 9 fasc., Simonkovich E. trad., 1965. Budapest, Hungary, Akademiai Kiado, p. 43-74.

24. MAVOUNGOU J.F., JAY-ROBERT P., GILLES J., ATSAME E.A., DUVALLET G., 2008. Ecologie des stomoxes (Diptera : Muscidae) au Gabon. I. Premier inventaire dans différentes zones écologiques. Parasite, $15:$ 27-34.

25. MAVOUNGOU J.F., MAKANGA B., ACAPOVI-YAO G., DESQUESNES M., M'BATCHI B., 2012. Chorologie des Tabanidae (Diptera) dans la réserve de biosphère Ipassa-Makokou (Gabon) en saison des pluies. Parasite, $19:$ 165-171.

26. MAVOUNGOU J.F., PICARD N., KOHAGNE L.T., M'BATCHI B., GILLES J., DUVALLET G., 2013. Spatio-temporal variation of biting flies, Stomoxys spp. (Diptera: Muscidae), along a man-made disturbance gradient, from primary forest to the city of Makokou (North-East, Gabon). Med. Vet. Entomol., 27: 339-345.
27. MIHOK S., 2002. The development of a multipurpose trap (the Nzi) for tsetse and other biting flies. Bull. Entomol. Res., 92: 385-403.

28. MIHOK S., KANG'ETHE E.K., KAMAU G.K., 1995. Trials of traps and attractants for Stomoxys spp. (Diptera: Muscidae). J. Med. Entomol., 32: 283-289.

29. NOIREAU F., NZOULANI A., SINDA D., ITOUA A., 1990. Chrysops silacea and C. dimidiata: fly densities and infection rates with Loa loa in the Chaillu mountains, Congo Republic. Trans. R. Soc. Trop. Med. Hyg., 84: 153-155.

30. OLDROYD H., 1952. The horse flies (Diptera: Tabanidae) of the Ethiopian region, Vol. I. London, UK, British Museum (Natural History), $226 \mathrm{p}$.

31. OLDROYD H., 1954. The horse flies (Diptera: Tabanidae) of the Ethiopian region, Vol. II. London, UK, British Museum (Natural History), $341 \mathrm{p}$.

32. OLDROYD H., 1957. The horse flies (Diptera: Tabanidae) of the Ethiopian region, Vol. III. London, UK, British Museum (Natural History), $489 \mathrm{p}$.

33. OLDROYD H., 1973. Tabanidae. In: Smith K.G.V. Ed., Insects and other arthropods of medical importance. London, UK, British Museum (Natural History), p. 195-202

34. R CORE TEAM, 2013. R: A language and environment for statistical computing. Vienna, Austria, R Foundation for Statistical Computing. www.R-project.org

35. RAYAISSE J.B., ESTERHUIZEN J., TIRADOS I., KABA D., SALOU E., DIARRASSOUBA A., VALE G.A., LEHANE M.J., TORR S.J., SOLANO P. 2011. Towards an optimal design of target for tsetse control: comparisons of novel targets for the control of Palpalis group tsetse in West Africa. PloS Negl. Trop. Dis., 5. DOI: 10.1371/journal.pntd.0001332

36. RAYAISSE J.B., KROBER T., MCMULLIN A., SOLANO P., MIHOK S., GUERIN P.M., 2012. Standardizing visual control devices for tsetse flies: West African species Glossina tachinoides, G. palpalis gambiensis and G morsitans submorsitans. PloS Negl. Trop. Dis., 6. DOI: 10.1371/journal. pntd.0001491

37. SURCOUF J., RICARDO G., 1909. Etude monographique des tabanides d'Afrique. Paris, France, Masson, 292 p.

38. VAN DE WEGHE J.P., 2006. Ivindo et Mwagna. Eaux noires, forêt vierge et baïs. Libreville, Gabon, Wildlife Conservation Society, $272 \mathrm{p}$.

39. WANJI S., TENDONGFOR N., ESUM M.E., ENYONG P., 2002 Chrysops silacea biting densities and transmission potential in an endemic area of human loiasis in south-west Cameroon. Trop. Med. Int Health, 7: 371-377.

40. ZINGA KOUMBA R.C., ACAPOVI YAO G.L., MAVOUNGOU J.F., TONGUE K., MBANG NGUEMA O.A., OBAME ONDO K.P., SHANGO M., 2013. Influence de la saison sur l'écodistribution des glossines, tabanides, stomoxes du Baï de Momba Makokou, Gabon. Agron. Afr., $25: 149-158$

41. ZUMPT F., 1973. The Stomoxyinae biting flies of the world (Diptera: Muscidae). Taxonomy, biology, economic importance and control measures. Stuttgart, Germany, Gustav Fischer Verlag, 175 p.

Accepté le 05.05.2014 


\section{Summary}

Zinga Koumba R.C., Bouyer J., Mavoungou J.F., Acapovi Yao G.L., Kohagne Tongué L., Mbang Nguema O.A., Ondo K.P.O., Mutambwe S. Assessment of the diversity of bloodsucking Diptera in a swampy forest clearing of Gabon with Vavoua and Nzi traps

The study aimed to assess the diversity of bloodsucking insects in the swampy forest clearings (bai) of Gabon with both Vavoua and Nzi traps. Twenty traps (10 of each type) were set up within and around Momba bai for seven consecutive days in June 2012. In each of the two biotopes, five pairs of traps (one Vavoua and one Nzi spaced about $30 \mathrm{~m}$ apart) were set at 250-400 m intervals. A total of 662 tsetse, 42 tabanids and 20 stable flies were collected. Vavoua traps captured many tsetse and stable flies, whereas Nzi traps caught more tabanids. Because of the short duration of capture, we later on pursued the investigation on the relative importance of these insects and their seasonal dynamics.

Keywords: Glossina - Stomoxys - Tabanidae - Vavoua trap Nzi trap - Forest - Swamp - Gabon.

\section{Resumen}

Zinga Koumba R.C., Bouyer J., Mavoungou J.F., Acapovi Yao G.L., Kohagne Tongué L., Mbang Nguema O.A., Ondo K.P.O., Mutambwe S. Evaluación de la diversidad de dípteros hematófagos en un claro de estero en Gabon, gracias a la ayuda de trampas Vavoua y Nzi

El objetivo del presente trabajo fue de estudiar la diversidad de insectos hematófagos presentes en los claros forestales de Gabon gracias a la ayuda de dos tipos de trampas: la Nzi y la Vavoua. Se colocaron veinte trampas (10 de cada tipo) al interior y alrededor del baï de Momba durante siete días consecutivos durante el mes de junio 2012. En cada uno de estos dos biotopos, cinco pares de trampas (una Vavoua y una Nzi distanciadas entre ellas de alrededor $30 \mathrm{~m}$ ) fueron colocadas a intervalos de 250-400 m. En total, se recolectaron 662 Glossinas, 42 tábanos y 20 Stomoxys. La trampa Vavoua capturó numerosos Stomoxys y Glossinas, mientras que la trampa Nzi capturó más tábanos. Considerando la corta duración de la captura, seguidamente se efectuó una prolongación del seguimiento de estos insectos para precisar su importancia relativa y su dinámica estacional.

Palabras clave: Glossina - Stomoxys - Tabanidae - Trampa Vavoua - Trampa Nzi - Bosque - Pantano - Gabón. 